
allemande

49-1 | 2017

Berlin 1957-1994

\title{
Gropiusstadt, Neukölln : un patronage prestigieux, une réputation sulfureuse
}

Hubert Guicharrousse

\section{(2) OpenEdition \\ 12 Journals}

Édition électronique

URL : https://journals.openedition.org/allemagne/525

DOI : 10.4000/allemagne.525

ISSN : 2605-7913

Éditeur

Société d'études allemandes

Édition imprimée

Date de publication : 16 juin 2017

Pagination : 85-98

ISSN : 0035-0974

Référence électronique

Hubert Guicharrousse, «Gropiusstadt, Neukölln : un patronage prestigieux, une réputation sulfureuse », Revue d'Allemagne et des pays de langue allemande [En ligne], 49-1 | 2017, mis en ligne le 16 juin 2018, consulté le 04 juin 2022. URL : http://journals.openedition.org/allemagne/525 ; DOI : https://doi.org/ 10.4000/allemagne.525 


\title{
Gropiusstadt, Neukölln: un patronage prestigieux, une réputation sulfureuse
}

\author{
- Hubert Guicharrousse*
}

La Gropiusstadt est un grand ensemble d'habitation construit entre 1962 et 1975, situé au sud-est de l'arrondissement de Neukölln, occupant une surface de 265 hectares. Avec 17000 logements et environ 35000 habitants $^{(1)}$, elle constitue la plus importante réalisation de ce type à Berlin-Ouest. L'association de ce grand ensemble au nom de Walter Gropius (1883-1969), fondateur du Bauhaus et «icône» de la modernité, ne manque pas d'intriguer. Quel fut donc le rôle de Gropius dans la conception de cette cité et quels sont les principes d'urbanisme qu'il a voulu lui appliquer? À quoi attribuer l'évolution du projet, de l'utopie urbanistique à sa réputation actuelle? Sa conception et sa réalisation doivent tout d'abord être replacées dans l'évolution urbaine de Berlin-Ouest et des politiques publiques du logement des années 50 à 70 . On présentera dans un deuxième temps les principales étapes de sa planification et de sa construction. Enfin, il y aura lieu d'esquisser l'évolution urbanistique et sociale de ce grand ensemble et de la confronter à sa réputation médiatique.

\section{Les grands ensembles dans la politique du logement à Berlin-Ouest}

\section{Démographie et crise du logement dans les années d'après-guerre}

La question du logement, dans le Berlin d'après-guerre, fut la préoccupation principale des autorités municipales. Comme dans toutes les villes allemandes gravement touchées par les bombardements, deux options s'offraient aux gouvernants, tant à l'Est qu'à l'Ouest: reconstruire l'espace urbain en respectant le plan préexistant ou réorganiser totalement la structure urbaine. À Berlin, plusieurs plans de réorganisation de la ville furent élaborés, dont le plus connu est le Kollektivplan de Hans Scharoun

* Maître de conférences à l'Université Paris-Ouest-Nanterre-La Défense.

1 Quartiersmanagement Gropiusstadt, http://www.qm-gropiusstadt.de/181.0.html. Le site Internet du Bezirksamt de Neukölln donne pour sa part 36842 habitants fin 2015: https://www.berlin.de/ ba-neukoelln/ueber-den-bezirk/zahlen-und-fakten/statistische-daten/einwohnerzahlen (sites consultés le 10 février 2017). 
(1893-1972), présenté en $1946^{(2)}$. Aucun de ces plans ne fut mis en œuvre, pour des raisons financières et techniques, ces dernières étant surtout liées à la présence d'infrastructures souterraines quasiment impossibles à modifier.

Dans une ville où plus du quart des logements avaient été endommagés par les bombardements, la crise du logement se posait avec une exceptionnelle gravité. La majeure partie de la population qui avait quitté la ville pendant la guerre retourna à Berlin après 1945. De très nombreux réfugiés vinrent s'ajouter à ce mouvement de convergence vers la capitale. La situation politique de l'après-guerre, en particulier le blocus soviétique des années 1948-49, ralentit la reconstruction de la partie occidentale de la ville, mais eut aussi pour résultat de gonfler le flux de réfugiés en provenance de la zone soviétique, même si la plupart d'entre eux étaient dirigés vers l'Allemagne de l'Ouest. De 1948 à 1958 (année de l'ultimatum de Khrouchtchev), la population de Berlin-Ouest augmenta de 125000 habitants, pour atteindre 2,26 millions, son étiage le plus élevé ${ }^{(3)}$. Confrontées à une pénurie de logements sans précédent, les autorités de Berlin-Ouest optèrent pour un développement très rapide du logement social, dont l'importance considérable devait rester une caractéristique jusqu'à l'unification.

\section{Le logement social et son financement}

Le Sénat de Berlin-Ouest, qui devait faire face à d'importantes difficultés financières, eut tout d'abord recours, dans les années 1949-1950, à des fonds du Plan Marshall (ERP), qui lui permirent de financer, avec des apports du gouvernement fédéral - le Berliner Aufbauprogramm -, principalement ses mesures d'urgence, dont l'objectif fut surtout de déblayer les décombres, mais aussi de construire une première série de logements sociaux. Environ 20 millions de DM de l'ERP furent ainsi utilisés dans les secteurs occidentaux de Berlin ${ }^{(4)}$. Aujourd'hui encore, les bâtiments construits à cette époque sont reconnaissables par la plaque métallique ornée d'un ours, indiquant l'année de réalisation.

De fait, la reconstruction de Berlin-Ouest resta tributaire des fonds fédéraux, dont la ville bénéficia dès l'entrée en vigueur, en avril 1950, de la Première loi sur la construction de logements (Erstes Wohnungsbaugesetz), portant sur le financement public du logement social. L'adoption par le Bundestag de cette loi marqua le véritable début de la reconstruction du parc de logements à Berlin-Ouest. En dépit des réserves exprimées par les trois gouverneurs militaires occidentaux en mai 1949, Berlin-Ouest fut de facto, dès la création du nouvel État, intégré au système des finances publiques de la RFA, comme en témoigne en particulier la Loi sur le soutien à l'économie du Grand Berlin (Ouest) du 7 mars 1950, rebaptisée en 1964 Loi sur l'aide à Berlin (Berlinhilfegesetz) ${ }^{(5)}$. L'un des premiers projets de construction financé dans ce cadre fut l'aménagement du secteur de l'actuelle station de métro de Britz-Süd à Neukölln, qui débuta en 1953, dans la continuité de la Cité du fer à cheval (Hufeisensiedlung), l'une des plus importantes réalisations du Berlin des années 20 en matière de logement social. Elle est principalement due à l'architecte Bruno

2 Greg Castillo, «Asceticism as Progress: Scharoun's Berlin Kollektivplan, 1946-1949», Journal of the Society of Architectural Historians, 73/3 (2014), p. 447-455.

3 Peter Ring, «Bevölkerung», in: Horst Ulrich, Uwe Prell (éd.), Berlin Handbuch. Das Lexikon der Bundeshauptstadt, Berlin, FAB Verlag, 1992, p. 240.

4 Wolfgang Bohleber, Mit Marshallplan und Bundeshilfe. Wohnungsbaupolitik in Berlin 1945 bis 1968, Berlin, Duncker \& Humblot, 1990, p. 135-136.

5 Gesetz zur Förderung der Wirtschaft von Groß-Berlin (West). Pour plus de précisions, cf. ibid., p. 179. 
Taut (1880-1938). Un autre projet important fut en 1957 la reconstruction de la partie sud du Hansaviertel dans l'arrondissement de Tiergarten, à laquelle participèrent des architectes internationaux tels Le Corbusier, Alvar Aalto, Oscar Niemeyer ou Walter Gropius, dans le cadre de l'Interbau 57 (quatrième édition de l'Exposition internationale d'architecture - Internationale Bauausstellung, IBA) ${ }^{(6)}$. Ce projet, conçu comme riposte occidentale à la construction de la Stalinallee, constitua le premier aménagement de grande ampleur réalisé à Berlin dans l'esprit de la Charte d'Athènes.

\section{Rénovation urbaine et Kahlschlagsanierung}

La crise du logement à Berlin-Ouest demeura une constante, et la construction du Mur en 1961 ne l'atténua pas. Même si la population des secteurs occidentaux ne cessa de diminuer après 1958 (jusqu'au milieu des années 80), l'offre de logements, au début des années 60 , restait très inférieure à la demande. La politique de rénovation du bâti ancien menée par le Sénat supprima dans un premier temps plus de surfaces habitables qu'elle n'en créa. L'habitat locatif berlinois traditionnel, celui des «casernes locatives » (Mietskasernen) de l'époque impériale, était critiqué depuis longtemps pour sa piètre qualité $^{(7)}$. En effet, le caractère très concentré de l'habitat, le manque d'équipements, les conditions de vie misérables, en particulier dans des quartiers comme Wedding, Kreuzberg ou Neukölln, conduisit les architectes et urbanistes de la mouvance dite du Neues Bauen, dès les années 1910, à proposer des solutions radicales de rénovation.

C'est au nom de ces carences urbaines que Willy Brandt, maire de Berlin depuis 1957, lança en 1963 le premier programme de rénovation urbaine (Erstes Stadterneuerungsprogramm), qui allait profondément bouleverser le paysage urbain de la capitale $^{(8)}$. Financé sur fonds fédéraux, ce programme, dont le but était de rénover 56000 logements considérés comme trop vétustes, conduisit en effet à la destruction pure et simple du bâti ancien, surtout dans les quartiers ouvriers de Wedding et Neukölln. Sur les terrains ainsi libérés, le Sénat, qui avait la plupart du temps fait jouer son droit de préemption, confia aux différentes sociétés de logements sociaux la construction de nouveaux immeubles. Ces modifications de très grande échelle, avec les aménagements visant à faire de Berlin-Ouest une "ville adaptée à l'automobile» (autogerechte Stadt), eurent des conséquences considérables. De nombreuses analyses critiques, dès cette époque, parlèrent même de "deuxième destruction " (après celles de la Deuxième Guerre mondiale).

Cette politique de rénovation urbaine, qui dura jusqu'à la fin des années 70 , fut systématique et sans la moindre considération pour le patrimoine architectural. La destruction de l'ancienne Mietkasernenstadt constituait en effet, aux yeux de ses promoteurs, un véritable bienfait. Ainsi, quand le bâtiment dit Meyer's-Hof à Wedding fut dynamité en 1970, personne n'éleva la voix. La destruction de quartiers entiers provoqua cependant plus tard un vaste mouvement de protestation, en particulier à

6 Sandra Wagner-Conzelmann, Die Interbau 1957 in Berlin, Petersberg, Michael Imhof Verlag, 2007.

7 Cf. Werner Hegemann, Das Steinerne Berlin. Geschichte der größten Mietskasernenstadt der Welt, Berlin, Kiepenheuer, 1930.

8 Sur ce programme, cf. Harald Bodenschatz et Cordelia Polinna, «Ein halbes Jahrhundert Stadterneuerung in Berlin», in: Günter Schlusche, Verena Pfeiffer-Kloss, Gabi Dolff-Bonek Ämper, Axel Klausmeier (éd.), Stadtentwicklung im doppelten Berlin. Zeitgenossenschaften und Erinnerungsorte, Berlin, Ch. Links Verlag, 2014, p. 206-213. 
Kreuzberg ${ }^{(9)}$ et Wedding. La politique de réhabilitation du Sénat, que les historiens de l'urbanisme qualifient de Kahlschlagsanierung, aggrava la crise du logement. Le terme de «Kahlschlag» utilisé dans la polémique berlinoise, est emprunté à la sylviculture et désigne la «coupe rase» ou «coupe claire», c'est-à-dire l'abattage d'arbres à grande échelle. L'incapacité à créer suffisamment de logements pour compenser des pertes considérables sur le marché locatif provoqua, au début des années 80 , le mouvement des squatteurs (Hausbesetzerbewegung).

En 1983, au cours de la mandature de Richard von Weizsäcker (1981-1984), le Sénat modifia sa politique en faveur d'une rénovation dite "prudente» ou «douce» (behutsame Renovierung). Ce tournant fut entériné par l'acceptation en mars 1983 par la Chambre des députés de Berlin des 12 principes de rénovation urbaine (12 Grundsätze der Stadterneuerung). Ces principes réclament une réhabilitation «douce», en particulier par la promotion du mélange des fonctions dans les centres urbains ${ }^{(10)}$. Les Expositions internationales d'architecture (IBA) organisées par le Sénat en 1984 et 1987 furent placées sous cette devise, qui constitua un véritable changement de paradigme dans l'aménagement urbain.

\section{Les grands ensembles à Berlin}

Dans les années 50 et 60 , tous les facteurs évoqués eurent pour résultat une raréfaction de l'offre de logements. La situation spécifique de la ville et donc l'impossibilité d'étendre les surfaces constructibles hors des limites des trois secteurs occidentaux fit très rapidement augmenter le prix des terrains. Il faut ajouter à cela l'urgence de trouver des solutions de relogement pour des milliers de locataires. L'option prise en faveur de la construction de grands ensembles (Großsiedlungen, Großwohnsiedlungen, Großwohnanlagen) en zone périphérique apparaît par conséquent parfaitement logique dans le contexte idéologique de l'époque. L'industrialisation des techniques de construction permettait en effet de construire rapidement, à grande échelle, et, en privilégiant la dimension verticale, de densifier l'habitat à peu de frais. À Berlin-Ouest, ce sont principalement 6 projets de grands ensembles qui furent réalisés ${ }^{(11)}$ :

\begin{tabular}{|l|l|c|c|}
\hline \multicolumn{1}{|c|}{ Nom de la cité } & Arrondissement/quartier & $\begin{array}{c}\text { Unités } \\
\text { d'habitation }\end{array}$ & $\begin{array}{c}\text { Années de } \\
\text { construction }\end{array}$ \\
\hline Gropiusstadt & Neukölln & 19000 & $1962 / 75$ \\
Märkisches Viertel & Reinickendorf & 17000 & $1963 / 73$ \\
Falkenhagener Feld & Spandau & 11000 & $1963 / 74$ \\
Hildburghauser Straße & Lichterfelde (Steglitz) & 5200 & $1964 / 74$ \\
Heerstraße-Nord & Spandau & 6800 & $1968 / 74$ \\
Thermometersiedlung & Lichterfelde (Steglitz) & 2700 & $1969 / 72$ \\
\hline \multicolumn{2}{|r|}{ Total des unités d'habitation: } & 61700 & \\
\hline
\end{tabular}

9 Voir au sujet de Kreuzberg, l'article de Patrick Farges dans ce même volume.

10 Sur les 12 Grundsätze, cf. Hardt-Waltherr HäMER, Behutsame Stadterneuerung, Berlin, Michael Bollé, Universität der Künste, 2007. On notera que la politique de la rénovation douce fut initiée par le même Hardt-Waltherr Hämer à partir du milieu des années 70, notamment avec le projet «Block 118 » à Charlottenburg.

11 Cf. Rainer BaAtz (éd.), Nachbesserungen von Groß-Siedlungen in Berlin (West) - Gropiusstadt, Berlin, Institut für Stadt- und Regionalplanung der Technischen Universität, Projektbericht 5, 1987, p. 5. 
Ces grands ensembles furent construits sur fonds publics, selon des principes inspirés de la Charte d'Athènes, publiée par Le Corbusier en 1941 sous le titre La Ville fonctionnelle (les principes en avaient été rédigés 10 ans auparavant). Le Corbusier et ses collègues du Congrès international d'architecture moderne (CIAM) de 1933 y préconisent notamment la division du tissu urbain en quatre zones, répondant aux «fonctions » suivantes: habitat, travail, loisirs, transports. Cette conception est en général appelée, dans le débat urbanistique allemand "die gegliederte und aufgelockerte Stadt » ${ }^{(12)}$, expression que l'on peut traduire par «ville structurée et aérée». En outre, la ville doit être divisée en «zones de voisinage» (Nachbarschaften) spécialisées dans l'une des quatre fonctions. C'est ce modèle qui présidera à la construction des grands ensembles dans les deux États allemands et bien sûr dans le reste de l'Europe et du monde.

\section{Planification et construction de la Gropiusstadt}

\section{La GEHAG et les quartiers sud de Neukölln: renouer avec les années 20?}

La Gropiusstadt est, en tout cas au départ, un projet de la Gemeinnützige Heimstätten-, Spar- und Bau-Aktiengesellschaft (GEHAG), société de logement social fondée en 1924 dans la mouvance syndicale, à l'origine de programmes de construction de l'entre-deux-guerres inspirés des cités-jardins anglaises qui ont fait la réputation de Berlin comme ville du logement social: Hufeisensiedlung (1925), Waldsiedlung Zehlendorf (1926), Onkel Toms Hütte (1926-1932), ainsi que la Wohnstadt Carl Legien à Prenzlauer Berg (1929). L'histoire de l'entreprise est étroitement liée au nom de Bruno Taut (1880-1938), qui en fut l'architecte principal et joua un rôle majeur dans l'élaboration de ces projets. Deux d'entre eux font partie des six cités d'habitation (Siedlungen der Berliner Moderne) classées en 2008 au patrimoine mondial de l'UNESCO. Le logo de la GEHAG figure d'ailleurs depuis 1927 sur le bâtiment en fer à cheval dû à Bruno Taut. Réorganisée en 1952, la GEHAG, avant sa privatisation à partir de 1998, appartenait au Land de Berlin, au DGB ainsi qu'à la Deutsche Angestellten-Gewerkschaft (DAG), qui détenaient chacun un tiers du capital ${ }^{(13)}$. Elle fut chargée en 1955 par le Sénat de construire un grand ensemble locatif. La logique des grands ensembles se heurtait cependant, dans le Berlin-Ouest de cette époque, au manque de terrains de grande étendue - contrairement à ce qui se passera dans les années 70-80 à Berlin-Est, où les grands ensembles de Marzahn et Hellersdorf bénéficièrent de surfaces considérables, de surcroît extensibles (comme ce fut le cas pour Hellersdorf).

La GEHAG identifia un territoire agricole d'environ 265 hectares, situé au sud de l'arrondissement de Neukölln, entre les villages de Britz, Buckow et Rudow. Elle projeta ainsi une extension de sa cité de Britz (Hufeisensiedlung). Dans l'esprit des responsables de la GEHAG, particulièrement de son directeur Karl-Heinz Peters, il s'agissait de renouer avec les cités de la modernité berlinoise qui avaient fait la réputation de la société dans les années 20 et 30. La planification et construction de ce qui s'appelait à l'origine BBR (pour Berlin-Buckow-Rudow) est l'une des mieux connues d'Allemagne.

12 Cette expression a été popularisée par Johannes Göderitz, Roland Rainer, Hubert Hoffmann, Die gegliederte und aufgelockerte Stadt, Tübingen, Wasmuth, 1957.

13 Site Internet de l'entreprise Deutsche Wohnen, à laquelle est aujourd'hui intégrée la GEHAG, https:// www.deutsche-wohnen.com/html/historie.php (site consulté le 5 février 2017). 
Une documentation complète et abondamment illustrée a été publiée en 1974 par les architectes Hans Bandel et Dittmar Machule ${ }^{(14)}$.

\section{Walter Gropius et la planification de la cité BBR}

La planification commença en 1958, en concertation avec le Sénat, et conduisit la GEHAG à procéder à des acquisitions de terrains. Jusqu'au début de la phase de construction, elle avait acquis à peu près la moitié de la surface totale (un quart étant propriété municipale). À la fin de l'année 1958, le directeur de la GEHAG émit l'idée de s'adresser à Walter Gropius pour réaliser les plans de la nouvelle cité. Gropius vivait depuis 1934 aux États-Unis, où il dirigeait un bureau d'architecture, TAC (The Architects Collaborative), sis à Cambridge (Massachusetts). L'idée de la GEHAG était de confier à Gropius la totalité du projet, tant le plan général que la conception des édifices, afin de ne pas répéter les erreurs de la reconstruction disparate du Hansaviertel. Architecte et urbaniste "progressiste» par excellence, le fondateur du Bauhaus était une légende vivante du Neues Bauen, mais aussi un architecte qui, depuis 1945, était revenu à plusieurs reprises dans sa ville natale et avait fourni plusieurs contributions à sa reconstruction. Il avait notamment participé à l'Interbau de 1957 en concevant avec son élève Wils Ebert (1909-1979) un bâtiment de neuf étages dans le Hansaviertel. Une demande officielle fut envoyée par la GEHAG en mars 1959 à Gropius, à laquelle l'intéressé répondit positivement. Wils Ebert, qui travaillait depuis 1945 pour l'administration de Berlin-Ouest et connaissait parfaitement la situation locale, fut nommé architecte de liaison par la GEHAG ${ }^{(15)}$. Le milieu professionnel des architectes de Berlin, cependant, souhaita très vite être partie prenante, afin de profiter de la manne des fonds fédéraux.

Le document de cadrage transmis par la GEHAG à Gropius prévoyait un projet de très grande ampleur: une cité comportant environ 16000 logements, où pourraient habiter 50000 personnes. Un tel ordre de grandeur n'avait alors jamais été atteint en Allemagne, et - si l'on excepte le Märkisches Viertel à Reinickendorf, d'une taille comparable - ne fut plus jamais atteint par la suite. Il s'agit donc bien là du point culminant de la construction de logements sur fonds publics en Allemagne. Les planificateurs ont dès le départ prévu un ensemble entièrement dédié au logement, conformément au principe de séparation fonctionnelle. Le directeur de la GEHAG Karl-Heinz Peters ne cessa d'ailleurs de répéter que rien n'était prévu sur le site pour des activités économiques ${ }^{(16)}$. Les trajets des futurs habitants jusqu'à leur lieu de travail devaient être facilités par la prolongation de la ligne de métro U7 de la station Grenzallee jusqu'à Rudow, promise par la société des transports publics de Berlin (BVG: Berliner Verkehrsbetriebe) dès le début du projet. Cette prolongation fut terminée en 1970, avant même l'achèvement

14 Hans Bandel, Dietmar Machule, Die Gropiusstadt. Der städtebauliche Planungs- und Entscheidungsvorgang, im Auftrag des Senators für Bau- und Wohnungswesen Berlin, Berlin, Kiepert, 1974. Voir également Roland Enke, «TAC 5431. Wie Berlin zur Gropiusstadt kam », in: Dorothea Kolland (éd.), Der lange Weg zur Stadt. Die Gropiusstadt im Umbruch, Berlin, Kramer, 2002, p. 7-18.

15 R. ENke, «TAC 5431 », ibid., p. 10.

16 Interview au Tagesspiegel du 16 janvier 1962: «Die neue Siedlung soll das Musterbeispiel einer Schlafstadt werden. Ein ganz ruhiges Wohngebiet, in dem kein Platz ist für Gewerbetreibende irgendwelcher Art. Ein Laden bringt für die Anwohner immer ein gewisses Maß an Belästigung mit sich. » Cité par Bandel/Machule, Die Gropiusstadt (note 14), p. 109. 
de la cité. La Gropiusstadt, avec ses quatre stations de métro, Johannisthaler Chaussee, Lipschitzallee, Wutzkyallee et Zwickauer Damm, est sans nul doute le grand ensemble allemand le mieux relié au réseau de transports local.

La GEHAG pria Gropius de fournir un plan d'aménagement (Strukturplan), ce qu'il fit en mai 1960 (1. TAC-Plan) ${ }^{(17)}$. Il prévoyait une voie verte (Grünzug) centrale située au sud du tracé de la ligne de métro, intégrant de nombreux équipements collectifs. 640 immeubles d'habitation étaient projetés, d'une hauteur maximale de 17 étages, les immeubles de quatre étages devant être les plus nombreux. Trois formes géométriques étaient prévues pour les constructions: cercle, rectangle et ligne. Le plan reçut un accueil extrêmement critique de la part de l'équipe de planification. On lui reprocha surtout de ne pas respecter le plan de voirie (Aufschließungsplan) fixé par la municipalité: l'axe central était beaucoup trop encombré d'équipements et ne respectait pas le principe de séparation des fonctions: il risquait de gêner les piétons. En outre, son orientation ne convenait pas au tracé de la ligne de métro. Enfin, les bureaucrates du Sénat critiquèrent l'utilisation trop systématique à leurs yeux de la forme circulaire, qui constituait une importante contrainte: ils souhaitaient la réserver à quelques points névralgiques comme les centres commerciaux ${ }^{(18)}$. Paradoxalement, toutes ces critiques étaient dirigées contre des éléments architecturaux que Gropius avait conçus comme des hommages à la Hufeisensiedlung et à Bruno Taut, dans la continuité desquels il était censé travailler. En réalité, le plan de voirie fixait par avance un grand nombre de paramètres: tracé du métro, des canalisations, situation des centres commerciaux, orientation de la voie verte, ainsi que la délimitation des surfaces à bâtir, bref tout ce qui incombe à une autorité de tutelle compétente en matière d'urbanisme et de construction, critères indispensables à l'obtention des fonds nécessaires à la construction. Gropius l'avait ignoré et était ainsi entré en conflit avec les autorités de sa ville natale.

\section{3. «Zu viele Köche verderben den Brei»}

La situation devint vite désagréable pour Gropius et ses collègues de TAC: le plan fut bel et bien rejeté - sans que le refus soit clairement exprimé. Il est vrai que le cabinet TAC n'avait guère l'expérience des projets de cette ampleur. Wils Ebert se rendit aux États-Unis avec un responsable de la GEHAG et présenta à Gropius toutes les critiques qui s'étaient exprimées. L'architecte accepta de revoir sa copie - sans enthousiasme. Peu après, la GEHAG signa avec TAC un contrat qui prévoyait des missions beaucoup plus limitées, comprenant un plan général de la cité respectant le plan d'aménagement du Sénat, ainsi que des plans de construction de quelques secteurs. Le deuxième plan (2. TAC-Plan) fut transmis à la GEHAG en avril 1961 et présenté par Gropius lui-même aux responsables berlinois. L'axe central y est remplacé par deux rues tangentielles, la voie verte est déplacée au nord de la ligne de métro, les accès piétons aux différents équipements sont améliorés. La densité des constructions est plus grande, et l'utilisation de la forme circulaire limitée (seulement trois constructions). Enfin, Gropius a prévu, à la demande de ses interlocuteurs, davantage d'espace pour le stationnement automobile. Cependant, l'architecte maintenait beaucoup de traits de son

17 Ibid., p. 36.

18 R. ENKE, «TAC 5431» (note 14), p. 12-13. 
premier plan, ce qui le rendait encombrant aux yeux d'une autorité de tutelle pressée par l'urgence. Il en résulta une isolation croissante de l'architecte, ce qui conduisit à son éviction de fait en tant que coordinateur du projet. Tout cela, bien sûr, resta dans le non-dit, car tant le Sénat que la GEHAG avaient besoin du nom de Gropius pour donner l'illusion que le projet renouait avec le passé glorieux des cités des années 20.

La période qui suivit, jusqu'au décès de Gropius en 1969, fut marquée par les malentendus et un manque de respect criant de l'équipe berlinoise envers l'architecte. La GEHAG salua le nouveau plan et considéra que la tâche de Gropius était achevée. Ce n'était manifestement pas l'avis de Gropius, qui revendiqua la supervision de la planification et de la construction jusqu'en 1966. En fait, la GEHAG avait déjà demandé au Sénat que la conception des bâtiments fût confiée à ses propres architectes. Cinq mois après la présentation du deuxième plan, Wils Ebert modifia une fois de plus le projet en tenant compte des demandes de densification supplémentaire formulées par le Sénat. Le projet échappait à Gropius et TAC, trop éloignés géographiquement et mentalement des réalités berlinoises. Gropius avait avant la guerre préconisé la verticalité (donc la tour d'habitation comme solution possible au problème du logement) mais devait à présent, de façon paradoxale, refréner les demandes de ses interlocuteurs visant à ajouter toujours plus d'étages aux bâtiments ${ }^{(19)}$.

Afin de faire profiter les architectes berlinois de ce projet gigantesque, le Sénat décida en mars 1962 d'impliquer, outre la GEHAG, six autres sociétés de construction de logements, dont la Degewo (Deutsche Gesellschaft zur Förderung des Wohnungsbaues, fondée en 1924), ainsi qu'une société privée d'investissement et sept maîtres d'ouvrage privés. Le terrain à bâtir devenait ainsi un patchwork de parcelles devant être loties de façon indépendante, sans coordination. La GEHAG chargea 14 architectes berlinois de faire des propositions pour les bâtiments prévus sur ses parcelles. En guise de lot de consolation, Gropius se vit confier le rôle d'expert dans la procédure de concours. Il se plia à cet exercice, tout en critiquant vivement la médiocrité des projets présentés et la disparité qu'on lui imposait ${ }^{(20)}$.

La Degewo adopta une procédure de concours analogue à la GEHAG et fit réaliser par Wils Ebert un plan de construction indépendant pour la partie est de la cité, dont elle avait la responsabilité. Walter Gropius, dans une lettre au directeur de la GEHAG, se plaignit de l'incompatibilité de ces procédures avec sa conception d'origine, soulignant que la ville moderne devait être une unité impliquant une coordination. Il exprima sa crainte de voir le projet dériver vers une addition de petites cités indépendantes, sans jonction (Nahtstellen) entre elles. Il se plaignit en outre du trop grand nombre de personnes impliquées dans le projet et de l'absence de communication et de coordination. Il ne cessa d'être mis devant le fait accompli, tant par la GEHAG que par le Sénat. En vain, il tenta de s'opposer à la densification imposée par l'administration, en particulier dans la partie est du site (21). $^{(2)}$

La densification supplémentaire apparut inévitable après la construction du Mur en août 1961. Ce qui devait à l'origine être une cité-jardin dans l'esprit de Bruno Taut

19 Bandel/Machule, Die Gropiusstadt (note 14), p. 114.

20 Ibid., p. 78.

21 Ibid., p. 114. 


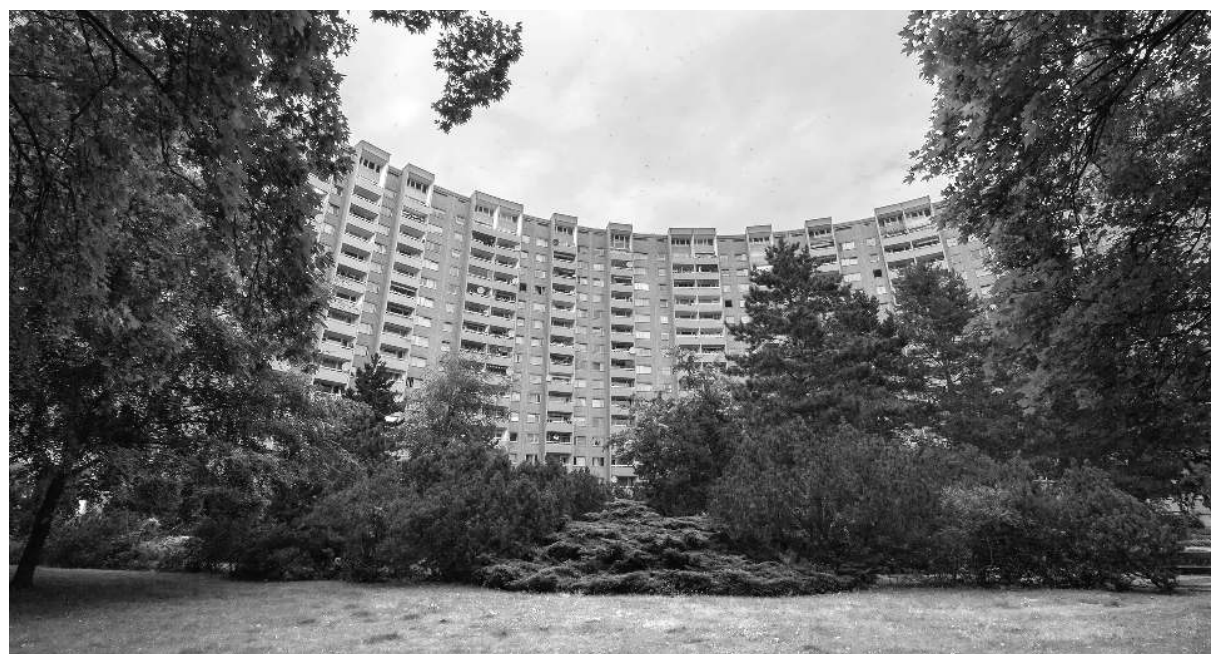

devint définitivement un grand ensemble. Le 7 novembre 1962, la première pierre fut solennellement posée en présence de Walter Gropius et Willy Brandt. Cependant, jusqu'à la fin de la construction, Gropius exprima à de nombreuses reprises ses critiques envers l'avancement du projet. Il ne réalisa au bout du compte avec TAC que les plans de trois bâtiments: la Gesamtschule qui porte aujourd'hui son nom, le bâtiment semi-circulaire dit Gropiushaus (ill. 1), enfin la tour IDEAL qui se trouve à proximité. Cette tour passe pour la plus haute tour d'habitation d'Allemagne (31 étages, $91 \mathrm{~m}$ ) et est située approximativement au centre de la cité (ill. 2). Après une dernière tentative institutionnelle en 1966 visant à obtenir le rôle de coordinateur «même sans rémunération », Gropius résuma ainsi son rôle dans la réalisation du projet: "Ich bin schließlich der zuletzt Verantwortliche für die Gesamtsiedlung und mir ist sehr daran gelegen, zu guten Ergebnissen zu kommen, aber wie Sie schreiben, scheint eine wirksame Koordination in der gegebenen Lage mit den vielen am Brei beteiligten Köchen äußerst schwierig zu sein ${ }^{(22)}$. Quelques mois plus tard, il tira même le bilan suivant:

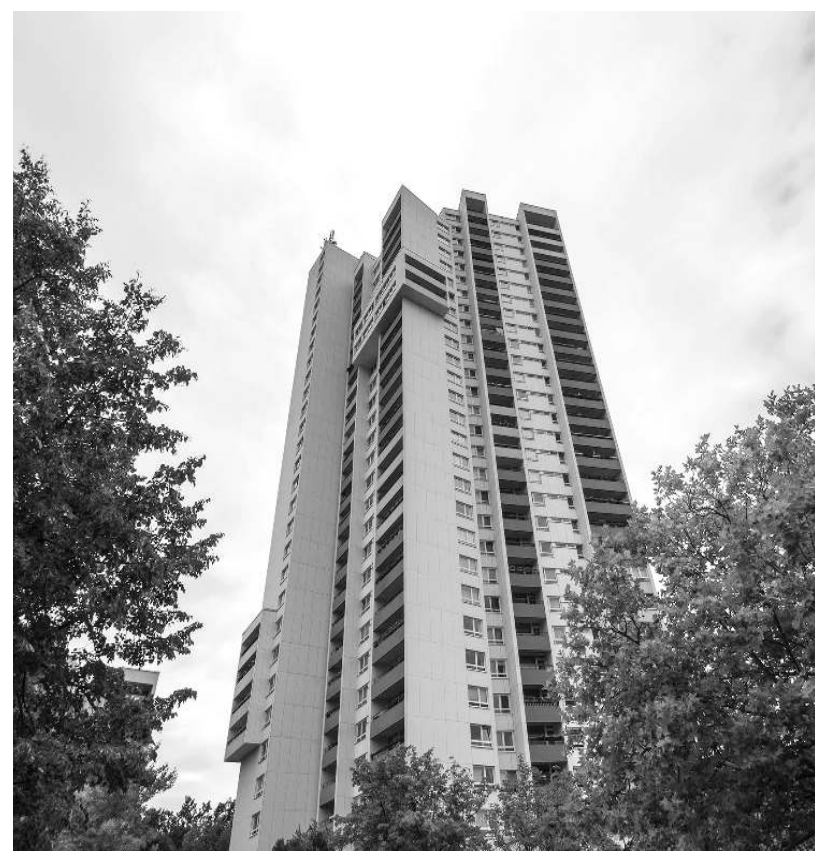

ill. 2

22 Lettre de W. Gropius à Hans Bandel, 10.01.1966, ibid., p. 112. 
«Ich muß gestehen, daß dieses Unternehmen das enttäuschendste ist, mit dem ich je zu tun hatte, da der Kontakt mit den Siedlungsträgergesellschaften fast auf den Nullpunkt gesunken ist ${ }^{(23)}$.

Trois ans après la mort de Gropius, en septembre 1972, la nouvelle cité fut officiellement baptisée Gropiusstadt, nom qui était déjà en usage avant cette décision mais qui, pour toutes les raisons exposées, tient de l'imposture. En effet, il ne reste plus grand-chose, dans l'actuelle cité, des conceptions initiales de Gropius, en particulier en ce qui concerne le nombre, la situation et surtout la hauteur des édifices, mais aussi les foyers de densification et les voies de communication. Au cours du travail de planification, les directives formulées par les responsables politiques, surtout après 1961, avaient été trop souvent modifiées, et les intérêts des différents acteurs étaient trop contradictoires pour permettre la réalisation d'un projet cohérent. Pour autant, la cité serait-elle devenue un lieu moins problématique si les idées de Gropius avaient été mises en œuvre dans le moindre détail? Il est bien sûr impossible de répondre à cette question. En revanche, il est légitime de se demander si les problèmes sociaux qui ont fait connaître la Gropiusstadt dans les médias peuvent être mis en relation avec son architecture et son urbanisme.

\section{Problèmes sociaux et urbanisme}

\section{L'effet Christiane F.}

«Gropiusstadt, das sind Hochhäuser für 45000 Menschen, dazwischen Rasen und Einkaufszentren. Von weitem sah alles neu und sehr gepflegt aus. Doch wenn man zwischen den Hochhäusern war, stank es überall nach Pisse und Kacke. Das kam von den vielen Hunden und den vielen Kindern, die in Gropiusstadt leben. Am meisten stank es im Treppenhaus» ${ }^{(24)}$.

C'est par ces mots que la biographie Christiane F. - Wir Kinder vom Bahnhof Zoo, publiée en 1978 par les journalistes Kai Hermann et Horst Rieck aux éditions du magazine Stern d'après des interviews, évoque la jeunesse de Christiane. Le film réalisé en 1981 par Ulrich Edel ${ }^{(25)}$ d'après le livre commence par deux phrases reprenant sous forme condensée cette description. On pourrait imaginer portrait plus flatteur que ce prologue dans lequel Christiane Felscherinow (puisque tel est son nom) évoque son enfance à la Gropiusstadt. La biographie, qui décrit une descente dans l'enfer de la toxicomanie et de la prostitution liée à la drogue, devint un best-seller mondial qui forgea très largement la réputation sulfureuse de la cité.

Les auteurs y évoquent assez longuement la situation des enfants livrés à eux-mêmes, délaissant les aires de jeu qui leur sont destinées, et dénoncent l'autoritarisme des concierges (Hauswarte) empêchant les enfants d'accéder à de très nombreux espaces. Résultat: les enfants entrent très vite en conflit avec le monde des adultes et forment des

23 Lettre de W. Gropius au sénateur chargé de l’Urbanisme, Werner Düttmann, 19 avril 1966, ibid., p. 112.

24 Christiane F., Kai Hermann, Horst Rieck, Christiane F. - Wir Kinder vom Bahnhof Zoo, nach Tonbandprotokollen aufgeschrieben von Kai Hermann und Horst Rieck, Vorwort von Horst E. RichTER, Hambourg, Stern-Verlag, 1978, p. 16. 
bandes qui les font dériver vers la délinquance. Face au manque chronique d'offre de loisirs destinée aux plus jeunes, les travailleurs sociaux sont impuissants ${ }^{(26)}$. Le contact avec la drogue commence pour Christiane à la Haus der Mitte, maison des jeunes de l'Église protestante (aujourd'hui fermée). Son premier responsable, le pasteur Jürgen Quandt, ne parvient pas à empêcher que la discothèque du centre ne devienne en un temps record une plaque tournante de la vente et consommation de drogues, d'abord douces, puis dures, suite à l'intrusion massive de l'héroïne dans le Berlin de la fin des années $60^{(27)}$.

L'évocation de l'enfance et de l'adolescence de Christiane Felscherinow établit un lien permanent entre l'architecture de la Gropiusstadt et surtout ses principes d'urbanisme et l'évolution des enfants et adolescents: absence d'espaces naturels utilisables, surfaces bétonnées reliant les constructions (ce qui empêche le développement de la vie sociale), dégradation très rapide des espaces intermédiaires, enfin constructions de piètre qualité, situation aggravée par un entretien inexistant. La Gropiusstadt est-elle pour autant plus touchée par les problèmes sociaux que d'autres cités du même genre en Allemagne, par exemple le Märkisches Viertel au nord de Berlin, Steilshoop à Hambourg ou encore Chorweiler à Cologne, pour ne citer que quelques lieux considérés par les médias comme des quartiers sensibles (soziale Brennpunkte)? On peut en douter. Pourtant, la Gropiusstadt reste attachée à Christiane F. - bien que l'histoire sociale de la cité ne se soit pas arrêtée à l'année 1978.

\section{2. "Améliorations» de l'habitat dans les années 80}

Confronté à de sévères critiques, le Sénat fut contraint, à l'occasion du $25^{\mathrm{e}}$ anniversaire de la cité, de prendre des mesures. Un comité consultatif fut créé, chargé de faire des propositions d'amélioration (Wohnumfeldverbesserungen) ${ }^{(28)}$. Outre des mesures de rénovation et d'embellissement des immeubles, on attacha une importance particulière aux espaces verts. En février 1986, un colloque fut organisé lors duquel des demandes diverses furent formulées, notamment au sujet de la rénovation du "paysage» de la cité, mais aussi des mesures de communication ayant pour but d'améliorer la relation entre les sociétés gestionnaires du parc immobilier et les habitants. Cependant, la question qui, aujourd'hui encore, constitue la critique principale des locataires, à savoir le montant des loyers, fut exclue de la discussion. Le Sénat consacra deux millions de DM à des mesures d'amélioration, essentiellement paysagères. En 1989, un conseil de quartier fut créé, dans lequel siègent, outre des représentants des habitants, des membres du conseil d'arrondissement de Neukölln et des représentants des sociétés de logement.

À la fin des années 80 et après l'unification, le Sénat continua à financer des mesures visant à améliorer l'offre de loisirs, pour les enfants et adolescents, mais aussi pour les personnes âgées. C'est ainsi que la Maison des jeunes et de la culture située près de la station de métro Wutzkyallee fut inaugurée en 1989. D’une façon générale, on peut dire que toutes ces mesures n'ont pas été vaines et ont globalement amélioré l'image de la cité. La Gropiusstadt dispose aujourd'hui d'une infrastructure sociale et médicale

Christiane F. (note 24), p. 24-34. 
qui n'a sans doute guère d'équivalent à Berlin et en Allemagne. L'engagement social du Sénat et des habitants de la cité est manifeste et a porté ses fruits. Depuis la réunification de Berlin, l'attention des médias s'est d'ailleurs plutôt focalisée sur les grands ensembles de la partie est (Marzahn et Hellersdorf), alors que ceux de l'Ouest sont moins soumis à la pression médiatique. Les "Plattenbauten» de la RDA sont ainsi devenus les nouveaux repoussoirs urbanistiques par excellence.

\section{3. Évolution sociale de la Gropiusstadt}

$\mathrm{Au}$ moment de l'achèvement de la cité, la population de la Gropiusstadt était de 45000 personnes, pour la plupart (environ $99 \%$ ) locataires des sociétés de logement social. Elle a aujourd'hui considérablement baissé, les habitants les plus favorisés ayant tendance à déménager. Les premiers locataires étaient surtout des employés, des cadres et des fonctionnaires, mais la composition sociale a beaucoup évolué depuis 1975, les sociétés gérantes ayant par la suite surtout attribué les logements à des ouvriers et à des chômeurs.

Dans les années 90, un apport de population très important a été constitué par des Allemands d'Europe de l'Est (Aussiedler, Spätaussiedler), surtout venus de Russie après l'éclatement de l'URSS. Le pourcentage d'étrangers est de $45 \%$, soit un peu plus que dans l'ensemble de l'arrondissement de Neukölln $(43 \%)^{(29)}$. Cette population est surtout concentrée dans la partie est. Pendant longtemps, la Gropiusstadt a eu un pourcentage d'étrangers de l'ordre de $10 \%$, soit beaucoup moins que le pourcentage de l'arrondissement. Cette augmentation est due principalement à un afflux considérable de population en provenance d'Europe de l'Est, après l'ouverture des frontières en 1990 et surtout depuis l'adhésion de nouveaux pays à l'Union européenne en 2004 et 2007. La proportion d'habitants d'origine turque ou arabe en revanche y est bien inférieure à celle de Neukölln, mais a tendance à augmenter, car les plus défavorisés d'entre eux migrent vers le sud de l'arrondissement. On constate aujourd'hui un fort contraste entre les habitants âgés, souvent des Allemands issus de la classe moyenne, qui ont constitué la première génération des années 60 et les nouveaux venus installés depuis l'unification, souvent étrangers et défavorisés. Le taux de chômage est supérieur à l'ensemble de l'arrondissement, qui atteint $15 \%$ (contre $11 \%$ sur l'ensemble de Berlin) ${ }^{(30)}$. Cependant, la Gropiusstadt se caractérise par une grande diversité de situations. Elle ne donne pas aujourd'hui l'impression du «désert de béton» souvent dénoncé dans les années 80 . La nature a eu le temps de s’y développer, et a profité des mesures d'embellissement paysager des années 80 et 90 . La Gropiusstadt est bien loin de la cité idéale, mais n'est manifestement pas non plus l'enfer évoqué par les médias.

Faut-il voir dans la malheureuse histoire de ce projet l'échec de l'urbanisme comme Gesamtkunstwerk, comme utopie d'un art considéré dans sa dimension créatrice, voire

29 Site Internet de l'arrondissement de Neukölln, consulté le 6 février 2017 - Über den Bezirk/Einwohnerregisterstatistik, URL: https://www.berlin.de/ba-neukoelln/ueber-den-bezirk/zahlen-und-fakten/ statistische-daten/einwohnerzahlen/.

30 Cf. Bezirksamt Neukölln von Berlin, Abteilung Jugend und Gesundheit, Planungs- und Koordinierungsstelle Gesundheit, Sozialbericht Neukölln. Zur sozialen Lage der Bevölkerung 2016, Berlin, janvier 2016. 
démiurgique, à qui l'on attribue le pouvoir de créer de nouvelles conditions sociales ou d'améliorer celles qui existent déjà? Ou ne faut-il pas plutôt raisonner en termes de rapports de forces, et constater l'échec de l'urbaniste-créateur face aux réalités sociales, économiques et politiques?

Dans le cas de la réalisation de la Gropiusstadt, on observe des institutions porteuses de projet (Trägergesellschaften) faiblement coordonnées, organisant volontairement, par souci de rapidité et de rentabilité, un éclatement architectural et urbanistique et finalement une disparité totale. En d'autres termes, on voit un mythe vivant de l'architecture et de l'urbanisme utilisé comme faire-valoir par les sociétés impliquées et l'autorité de tutelle, afin de créer très rapidement et à des coûts les plus bas possibles un grand nombre de logements, sans se poser la question des conséquences de la densification ainsi entreprise.

Et pourtant, il faut noter que, dans le débat qui a lieu à Berlin depuis plusieurs années sur le thème du logement "abordable» (bezahlbarer Wohnraum), la construction de tours d'habitation et de grands ensembles redevient une hypothèse plausible. Dans une ville dont la population augmente d'environ 40000 personnes par an, on annonce ces derniers mois non seulement la construction d'une tour sur l'Alexanderplatz, mais aussi de nouveaux grands ensembles, notamment à Lichterfelde et Pankow. Une fois encore, ces projets se feront dans l'urgence. Faut-il redouter que les erreurs commises il y a cinquante ans se répètent?

\section{Bibliographie}

Rainer BAATz (éd.), Nachbesserungen von Groß-Siedlungen in Berlin (West) - Gropiusstadt, Berlin, Institut für Stadt- und Regionalplanung der Technischen Universität, Projektbericht 5, $1987,88 \mathrm{p}$.

Hans Bandel, Dietmar Machule, Die Gropiusstadt. Der städtebauliche Planungs- und Entscheidungsvorgang, im Auftrag des Senators für Bau- und Wohnungswesen Berlin, Berlin, Kiepert, 1974, 178 p.

Bezirksamt Neukölln von Berlin, Abteilung Jugend und Gesundheit, Planungs- und Koordinierungsstelle Gesundheit, Sozialbericht Neukölln. Zur sozialen Lage der Bevölkerung 2016, Berlin, janvier 2016, 38 p.

Harald Bodenschatz et Cordelia Polinna, «Ein halbes Jahrhundert Stadterneuerung in Berlin», in: Günter Schlusche, Verena Pfeiffer-Kloss, Gabi Dolff-Bonekëmper, Axel KLAusmeier (éd.), Stadtentwicklung im doppelten Berlin. Zeitgenossenschaften und Erinnerungsorte, Berlin, Ch. Links Verlag, 2014, p. 206-213.

Wolfgang Bohleber, Mit Marshallplan und Bundeshilfe. Wohnungsbaupolitik in Berlin 19451968, Berlin, Duncker \& Humblot, 1990, 310 p.

Greg Castillo, «Asceticism as Progress: Scharoun's Berlin Kollektivplan, 1946-1949», Journal of the Society of Architectural Historians, 73/3 (2014), p. 447-455.

Roland Enke, «TAC 5431. Wie Berlin zur Gropiusstadt kam», in: Dorothea Kolland (éd.), Der lange Weg zur Stadt. Die Gropiusstadt im Umbruch, Berlin, Kramer, 2002, p. 7-18.

Christiane F., Kai Hermann, Horst Rieck (éd.), Christiane F. - Wir Kinder vom Bahnhof Zoo, nach Tonbandprotokollen aufgeschrieben von Kai Hermann und Horst Rieck und mit einem Vorwort von Horst E. Richter, Hambourg, Stern-Verlag, 1978, 333 p.

Johannes Göderitz, Roland Rainer, Hubert Hoffmann, Die gegliederte und aufgelockerte Stadt, Tübingen, Wasmuth, 1957, $101 \mathrm{p}$.

Hardt-Waltherr HäMER, Behutsame Stadterneuerung, Berlin, Universität der Künste, 2007, 95 p. 
Werner Hegemann, Das Steinerne Berlin. Geschichte der größten Mietskasernenstadt der Welt, Berlin, Kiepenheuer, 1930, 505 p.

Peter Ring, «Bevölkerung», in: Horst Ulrich, Uwe Prell (éd.), Berlin Handbuch. Das Lexikon der Bundeshauptstadt, Berlin, FAB Verlag, 1992, 1548 p.

Sandra Wagner-Conzelmann, Die Interbau 1957 in Berlin, Petersberg, Michael Imhof Verlag, 2007, $192 \mathrm{p}$.

\section{Résumé}

La Gropiusstadt, grand ensemble de Berlin-Ouest, porte un nom prestigieux. Mais quel fut réellement le rôle du créateur du Bauhaus dans la conception et la réalisation de cette cité située au sud de l'arrondissement de Neukölln? Plus largement, pourquoi le Sénat de Berlin-Ouest opta-t-il pour la construction de grands ensembles? La réponse à ces questions conduit à replacer la construction de la Gropiusstadt dans le contexte des politiques d'urbanisme des années 50 à 80, en particulier comme conséquence de la "rénovation urbaine" mise en cuvre par le maire de Berlin Willy Brandt à partir de 1963. La mauvaise réputation faite à la cité depuis la fin des années 70 doit d'autre part être confrontée à la réalité de son évolution sociale.

\section{Zusammenfassung}

Die West-Berliner Großsiedlung Gropiusstadt trägt einen prestigeträchtigen Namen. Welche Rolle spielte der Begründer des Bauhaus in der Planung und Verwirklichung dieser Siedlung im Süden des Bezirks Neukölln? Warum optierte darüber hinaus der West-Berliner Senat für den Bau von Großsiedlungen? Die Beantwortung dieser Fragen führt dazu, den Bau der Gropiusstadt im Kontext der Stadtpolitik der Fünfzigerbis Achtzigerjahre zu betrachten, insbesondere als Folge der von Bürgermeister Willy Brandt seit 1963 umgesetzten "Stadterneuerung». Ferner wird der schlechte Ruf, der seit Ende der Siebzigerjahre der Siedlung vorauseilt, mit der Realität ihrer sozialen Entwicklung konfrontiert. 